\title{
X Chromosome Evolution in Cetartiodactyla
}

\author{
Anastasia A. Proskuryakova 1,2,*, Anastasia I. Kulemzina ${ }^{1}$, Polina L. Perelman 1,2 (D), \\ Alexey I. Makunin ${ }^{1}$, Denis M. Larkin ${ }^{3}$ (D), Marta Farré ${ }^{3}$ (D), Anna V. Kukekova ${ }^{\text {, }}$ \\ Jennifer Lynn Johnson ${ }^{4}$, Natalya A. Lemskaya ${ }^{1}$, Violetta R. Beklemisheva ${ }^{1}$, \\ Melody E. Roelke-Parker ${ }^{5}$, June Bellizzi ${ }^{6}$, Oliver A. Ryder ${ }^{7}$, Stephen J. O'Brien ${ }^{8,9}$ and \\ Alexander S. Graphodatsky ${ }^{1,2}$ \\ 1 Institute of Molecular and Cellular Biology, SB RAS, Lavrentiev Ave. 8/2, Novosibirsk 630090, Russia; \\ zakal@mcb.nsc.ru (A.I.K.); polina.perelman@gmail.com (P.L.P.); alex.makunin@gmail.com (A.I.M.); \\ lemnat@mcb.nsc.ru (N.A.L.); bekl@mcb.nsc.ru (V.R.B.); graf@mcb.nsc.ru (A.S.G.) \\ 2 Synthetic Biology Unit, Novosibirsk State University, Pirogova Str. 1, Novosibirsk 630090, Russia \\ 3 The Royal Veterinary College, University of London, Royal College Street, London NW1 0TU, UK; \\ dmlarkin@gmail.com (D.M.L.); mfarrebelmonte@gmail.com (M.F.) \\ 4 Animal Sciences Department, College of ACES, University of Illinois at Urbana-Champaign, IL 61801, USA; \\ avk@illinois.edu (A.V.K.); jjohnso@illinois.edu (J.L.J.) \\ 5 Frederick National Laboratory of Cancer Research, Leidos Biomedical Research, Inc., Frederick, MD 21702, \\ USA; melodyr@mail.nih.gov \\ 6 Catoctin Zoo and Wildlife Preserve, Thurmont, MD 21788, USA; rapunta@yahoo.com \\ 7 San Diego Zoo Institute for Conservation Research, 15600 San Pasqual Valley Road, Escondido, CA 92027, \\ USA; oryder@sandiegozoo.org \\ 8 Theodosius Dobzhansky Center for Genome Bioinformatics, Saint-Petersburg State University, \\ Sredniy Av. 41A, Saint-Petersburg 199034, Russia; lgdchief@gmail.com \\ 9 Oceanographic Center, Nova Southeastern University, Fort Lauderdale 3301 College Ave, Fort Lauderdale, \\ FL 33314, USA \\ * Correspondence: andrena@mcb.nsc.ru; Tel.: +7-960-799-5653
}

Academic Editor: Thomas Liehr

Received: 1 August 2017; Accepted: 25 August 2017; Published: 31 August 2017

\begin{abstract}
The phenomenon of a remarkable conservation of the $\mathrm{X}$ chromosome in eutherian mammals has been first described by Susumu Ohno in 1964. A notable exception is the cetartiodactyl $\mathrm{X}$ chromosome, which varies widely in morphology and G-banding pattern between species. It is hypothesized that this sex chromosome has undergone multiple rearrangements that changed the centromere position and the order of syntenic segments over the last 80 million years of Cetartiodactyla speciation. To investigate its evolution we have selected 26 evolutionarily conserved bacterial artificial chromosome (BAC) clones from the cattle CHORI-240 library evenly distributed along the cattle $\mathrm{X}$ chromosome. High-resolution BAC maps of the $\mathrm{X}$ chromosome on a representative range of cetartiodactyl species from different branches: pig (Suidae), alpaca (Camelidae), gray whale (Cetacea), hippopotamus (Hippopotamidae), Java mouse-deer (Tragulidae), pronghorn (Antilocapridae), Siberian musk deer (Moschidae), and giraffe (Giraffidae) were obtained by fluorescent in situ hybridization. To trace the $\mathrm{X}$ chromosome evolution during fast radiation in specious families, we performed mapping in several cervids (moose, Siberian roe deer, fallow deer, and Pere David's deer) and bovid (muskox, goat, sheep, sable antelope, and cattle) species. We have identified three major conserved synteny blocks and rearrangements in different cetartiodactyl lineages and found that the recently described phenomenon of the evolutionary new centromere emergence has taken place in the $\mathrm{X}$ chromosome evolution of Cetartiodactyla at least five times. We propose the structure of the putative ancestral cetartiodactyl X chromosome by reconstructing the order of syntenic segments and centromere position for key groups.
\end{abstract}


Keywords: Pecora; Ruminantia; cattle bacterial artificial chromosome (BAC) clones; fluorescent in situ hybridization (FISH); intrachromosomal rearrangements; centromere reposition; inversion

\section{Introduction}

Despite the great variation in diploid number and high level of autosome reshuffling, the $X$ chromosome of eutherian mammals is evolutionary conserved. The size and morphology of the $\mathrm{X}$ chromosome as defined by the position of the centromere is similar in most mammalian orders. Hypothetically, this unique conservation was guided by the establishment of a mechanism for dosage compensation in the therian ancestor [1]. The emergence of this mechanism is thought to have imposed evolutionary constraints on chromosomal rearrangements in the sex chromosome [1].

Classical cytogenetic techniques were used to describe morphology, centromere position, banding pattern, and heterochromatin distribution in a wide range of species. Comparative analysis has identified similar $X$ chromosome morphology and G-banding patterns across species from different taxa (primates, pigs, camels, carnivores, perissodactyls) [2]. Comparative mapping of the $\mathrm{X}$ chromosome with gene-specific probes confirmed similarity in the gene order on the $\mathrm{X}$ chromosome of distantly related species (human, pig, horse, dog, cat) [3]. These studies provided strong evidence for Ohno's rule, confirming genomic conservancy of eutherian $\mathrm{X}$ chromosomes. However, some notable exceptions in conservation phenomenon of $X$ chromosome have been identified in Cetartiodactyla and Rodentia. The modified $\mathrm{X}$ chromosome structure in these orders is caused by inversions, changes in centromere position, heterochromatin expansion and autosome to sex chromosome translocations [4].

The order Cetartiodactyla exhibits great diversity of chromosome X morphology both within and between families. Note that in most eutherian orders only autosomal syntenic segments undergo reshuffling as shown by cross-species chromosome painting [5]. The exact mechanisms behind dynamic changes on $\mathrm{X}$ chromosome in Cetartiodactyla are unknown. Comparative chromosome painting with whole chromosome painting probes, including $X$, has been employed in several studies [6-11]. These studies showed that cetartiodactyl autosomes evolved through fission, fusion, and inversions. However, unlike autosomes, the sex chromosomes evolved through more complex chromosomal rearrangements involving reshuffling of conserved segments inside the chromosome, changes in centromere positions, heterochromatic variation, and autosomal translocations [12,13]. It is likely that centromere repositioning (shift) or so-called evolutionary new centromere phenomenon, reflecting a change of centromere position on the chromosome without a change in the gene order, also occurred in cetartiodactyl X chromosome evolution. So far it was shown only in primates, rodents and perissodactyls [14-17].

The structure of cetartiodactyl $\mathrm{X}$ chromosomes has been closely studied mainly in domestic species from the family Bovidae [13,18-22], and in a few wild species from the families Giraffidae, Cervidae, Antilocapridae and Hippopotamidae [6,23-25]. In previous studies, microdissection probes or arm-specific paints and several bacterial artificial chromosome (BAC) clones were used to detect intrachromosomal rearrangements. A recent investigation showed centromere repositioning and inversions in cetartiodactyl $X$ chromosomes [25]. Interspecific $X$ chromosome variation in the Cetartiodactyla has been a source of some controversy in the past [12]. The analysis of $X$ chromosome rearrangements can be a potential source of phylogenetic information [12], but the $\mathrm{X}$ chromosome evolution in Cetartiodactyla has not yet been studied in detail.

In the present study, we report the comparative map of cetartiodactyl X chromosomes obtained by cross-species hybridization with the set of cattle BAC clones, and provide new data about $\mathrm{X}$ chromosome evolution in 10 cetartiodactyl families. Our analysis allows reconstruction of the ancestral $\mathrm{X}$ chromosome for major nodes of the cetartiodactyl tree and traces the rearrangements of $\mathrm{X}$ chromosome that have occurred during evolution within this order. 


\section{Materials and Methods}

\subsection{Species}

The list of studied species with scientific and common names, diploid chromosome number, and source of cell lines is presented in the Table 1. All cell lines belong to the cell cultures collection of general biological purpose (No. 0310-2016-0002) of Institute of Molecular and Cellular Biology Siberian Branch of the Russian Academy of Sciences.

Table 1. List of cetartiodactyl species included in this study and their characteristics.

\begin{tabular}{|c|c|c|c|c|c|}
\hline $\begin{array}{l}\text { Scientific Name, } \\
\text { Abbreviation }\end{array}$ & Code & Common Name & Family & $\begin{array}{l}\text { Diploid } \\
\text { Number }\end{array}$ & Source of Cell Line \\
\hline Sus scrofa & SSC & Pig & Suidae & $38, X X$ & IMCB SB RAS, Novosibirsk-1* \\
\hline Lama pacos & LPA & Alpaca & Camelidae & $74, X Y$ & $2^{*}$ \\
\hline Eschrihtius robustus & ERO & Gray whale & Eschrichtiidae (Cetacea) & $44, X Y$ & [11] \\
\hline Hippopotamus amphibius & HAM & Common hippopotamus & Hippopotamidae & $36, X Y$ & [8] \\
\hline Tragulus javanicus & TJA & Java mouse-deer & Tragulidae & $32, X Y$ & $\begin{array}{c}\text { Frozen Zoo (San Diego Zoo's Conservation } \\
\text { Research, San Diego, CA, USA) }\end{array}$ \\
\hline Antilocapra americana & AAM & Pronghorn & Antilocapridae & $58, X Y$ & [10] \\
\hline Giraffa camelopardalis & GCA & Giraffe & Giraffidae & $30, X Y$ & [8] \\
\hline Moschus moschiferus & $\mathrm{MMO}$ & Siberian musk deer & Moschidae & $58, X Y$ & [8] \\
\hline Dama dama & DDA & Fallow deer & Cervidae, Cervinae & $68, x X$ & $\begin{array}{c}\text { Catoctin Wildlife Preserve and Zoo, } \\
\text { Maryland, USA }\end{array}$ \\
\hline Elaphurus davidianus & EDA & Pere David's deer & & $68, X X$ & $3^{*}$ \\
\hline Alces alces & AAL & Eurasian elk & Cervidae Canreolinae & $68, X X$ & IMCB SB RAS, Novosibirsk \\
\hline Capreolus pygargus & CPY & Siberian roe deer & Cervidae, Capreolinae & $70, X X$ & IMCB SB RAS, Novosibirsk \\
\hline Ovibos moschatus & $\mathrm{OMO}$ & Muskox & & $48, \mathrm{XX}$ & IMCB SB RAS, Novosibirsk \\
\hline Capra hircus & CHI & Goat & Bovidae, Antilopinae & $60, X X$ & $\begin{array}{c}\text { Catoctin Wildlife Preserve and Zoo, } \\
\text { Maryland, USA }\end{array}$ \\
\hline Ovis aries musimon & OAR & Sheep & & $54, \mathrm{XX}$ & $\begin{array}{c}\text { Catoctin Wildlife Preserve and Zoo, } \\
\text { Maryland, USA }\end{array}$ \\
\hline Hippotragus niger & HNI & Sable antelope & & $60, \mathrm{XX}$ & $3^{*}$ \\
\hline Bison bison & BBI & American bison & Bovidae Bovinae & $60, \mathrm{XX}$ & $4^{*}$ \\
\hline Bos taurus & BTA & Cattle & Bovidae, Bovinae & $60, X X$ & IMCB SB RAS, Novosibirsk \\
\hline
\end{tabular}

$1^{*}$ : IMCB SB RAS - Institute of Molecular and Cellular Biology Siberian Branch of the Russian Academy of Sciences. 2*: The cell line is established by William Nash (Laboratory of Genomic Diversity, NCI, Frederick, MD, USA). The sample provided by Camelid Research Group (Oregon State University, Corvallis, OR, USA). 3*: Sample provided by Mitchell Bush (Conservation and Research Center, National Zoological Park, Front Royal, VA, USA). Cell line is established in the Laboratory of Genomic Diversity (NCI, Frederick, MD, USA). $4^{*}$ : The sample is provided by Douglas Armstrong (Henry Doorly Zoo, Omaha, NE, USA). Cell line is established in the Laboratory of Genomic Diversity (NCI, Frederick, MD, USA).

\subsection{Chromosome Preparation}

Metaphase chromosomes were obtained from fibroblast cell lines. Briefly, cells were incubated at $37{ }^{\circ} \mathrm{C}$ and $5 \% \mathrm{CO}_{2}$ in medium $\alpha \mathrm{MEM}$ (Sigma Aldrich Co., St. Louis, MO, USA) supplemented with 15\% fetal bovine serum, 5\% AmnioMAX-II complete (Gibco ${ }^{\mathrm{TM}}$ ) and antibiotics (ampicillin $100 \mu \mathrm{g} / \mathrm{mL}$, penicillin $100 \mu \mathrm{g} / \mathrm{mL}$, amphotericin B $2.5 \mu \mathrm{g} / \mathrm{mL}$ ). Metaphases were obtained by adding colcemid $(0.02 \mathrm{mg} / \mathrm{mL})$ and ethidium bromide $(1.5 \mathrm{mg} / \mathrm{mL})$ to actively dividing culture for $3-4 \mathrm{~h}$. Hypotonic treatment was performed with $3 \mathrm{mM} \mathrm{KCl}, 0.7 \mathrm{mM}$ sodium citrate for $20 \mathrm{~min}$ at $37^{\circ} \mathrm{C}$ and followed by fixation with 3:1 methanol/glacial acetic acid (Carnoy's) fixative. Metaphase chromosome preparations were made from a suspension of fixed fibroblasts, as described previously [26]. G-banding on metaphase chromosomes prior to fluorescence in-situ hybridization (FISH) was performed using standard procedure [27].

\subsection{BAC Clones}

Using the cattle genome assembly version from October 2011 (Baylor Btau_4.6.1/bosTau7) in UCSC Genome Browser [28], X chromosome-located BAC clones were manually chosen from the CHORI-240 BAC library from the "BACPAC Resource Center" (BPRC, the Children's Hospital Oakland Research Institute in Oakland, CA, USA). To download information in the Genome Browser about the localization of BACs of appropriate size (length of insertion varied from 50-300 kb), a custom track in Browser Extensible Data (BED) format was created [29]. BAC clones with appropriate insert sizes (50-300 kbp) and genetic content (unique genes, less repetitive elements) were selected. BAC sequence 
conservation was estimated from phyloP data [30] in the human genome ("Conservation" track in GRCh37/hg19 assembly). Genome coordinates were converted from cow to human using the Batch Coordinate Conversion (liftOver tool) in UCSC Genome Browser. Thus, 73 BAC clones evenly distributed on cattle $X$ chromosome (2-5 Mbp gaps) were selected. For each of the manually selected 73 BACs, we defined various genomic features selected to increase the probability of a clone to hybridize with metaphase spreads of distant cetartiodactyl species. To do so, we calculated protein coding genes, cattle genes orthologous to human, GC content, and repetitive sequences in each of the selected BAC clones. By using multiple alignments, including all available cetartiodactyl genomes, we calculated the nucleotide conservation scores and conserved elements using phastCons [31]. Then, we compared the characteristics of four BACs that had previously worked on distant species with all the 73 BACs by using the classification tree from the CART algorithm [32]. A total of 51 BACs were selected to have a high probability of hybridization to distant species. These BACs contained less than $48 \%$ of repetitive sequence and more than $20 \%$ of conserved elements. A subset of 26 of these BAC clones that were evenly distributed along the cattle $\mathrm{X}$ chromosome with a median distance of $5 \mathrm{Mb}$ were hybridized on all cetartiodactyl species studied here. Table 2 lists the CHORI-240 cattle $X$ chromosome BAC clones used in this study. A single BAC clone (CH240-316D2) is the same as used by Fröhlich et al. [25].

BAC DNA was isolated using the Plasmid DNA Isolation Kit (BiosSilica, Novosibirsk, Russia) and amplified with GenomePlex Whole Genome Amplification kit (Sigma-Aldrich Co., St. Louis, MO, USA). Labeling of BAC DNA was performed using GenomePlex WGA Reamplification Kit (Sigma-Aldrich Co., St. Louis, MO, USA) by incorporating biotin-16-dUTP or digoxigenin-dUTP (Roche, Basel, Switzerland). The quality of produced BAC probes was controlled by FISH localization on cattle chromosomes.

\subsection{Fluorescence In-Situ Hybridization (FISH)}

Dual-color FISH experiments on G-banded metaphase chromosomes were performed as described by Yang and Graphodatsky [26]. Digoxigenin-labeled and biotin-labeled probes were detected with $\mathrm{Cy}^{\mathrm{TM}_{3}}$ anti-digoxin (Jackson ImmunoResearch Laboratories, Inc., West Grove, PA), fluorescein avidin DCS, biotinilated anti-avidin D (Vector Laboratories, Inc., Burlingame, CA, USA), respectively. Images were captured with a Baumer Optronics CCD Camera (Baumer Ltd., Southington, CT, USA) mounted on an Olympus BX53 microscope (Olympus, Shinjuku, Japan) and processed using VideoTesT 2.0 Image Analysis System (Zenit, St. Petersburg, Russia).

\subsection{Bioinformatics Analysis}

An analysis in UCSC Genome Browser was performed to establish the order of CHORI-240 BAC clones on $X$ chromosomes of one cetartiodactyl species (sheep) and four species from out-group mammalian orders (Perissodactyla, Primates, Rodentia). BAC positions in these genomes were obtained using Batch Coordinate Conversion (liftOver) in the UCSC Genome Browser that converts genome coordinates between assemblies. The cattle genome assembly (Bos_taurus_UMD3.1.1/bosTau8) was used as a reference. Sequences coordinates of all BAC clones were calculated in human (GR ch38/hg 38), mouse (GRC m38/mm10), rat (RGSC 6.0/rn6, except 386M8, which is disrupted in this genome), horse (Broad/equCab2), and sheep (ISGC Oar_v3.1/oviAri3) genomes.

\subsection{Ancestral Chromosome Deduction}

The morphology and conservative block orientation of the ancestral $X$ chromosome were deduced using maximum parsimony by comparing $X$ chromosomes across the top branches of Cetartiodactyla and assuming the most common variant to be ancestral for the order. Once the provisional ancestral chromosome was identified, we detected whether the extant $X$ chromosome and the suggested ancestral form differ by inversions (change of BAC order) or/and by centromere repositioning (change of centromere position without change in BAC order). 
Table 2. CHORI-240 BAC's order on cetartiodactyl X chromosomes. The color of the cells corresponds to a certain conservative syntenic segment.

\begin{tabular}{|c|c|c|c|c|c|c|c|c|c|c|c|c|c|c|c|c|c|c|}
\hline \multirow[b]{2}{*}{ No. } & \multirow{2}{*}{\multicolumn{3}{|c|}{$\begin{array}{l}\text { BAC's Order and Localization on } \\
\text { Cattle X chromosome }\end{array}$}} & \multicolumn{15}{|c|}{ CHORI (CH-240) BACs Localization on Cetartiodactyl X chromosomes } \\
\hline & & & & $\begin{array}{l}\text { Domestic } \\
\text { Pig, sSC }\end{array}$ & $\begin{array}{c}\text { Alpaca, } \\
\text { LPA }\end{array}$ & $\begin{array}{c}\text { Gray Whale, } \\
\text { ERO }\end{array}$ & $\begin{array}{c}\text { Common Hippopota- } \\
\text { mus, HAM }\end{array}$ & $\begin{array}{l}\text { Java Mouse- } \\
\text { Deer, TJA }\end{array}$ & $\begin{array}{l}\text { Pronghorn, } \\
\text { AAM }\end{array}$ & $\begin{array}{c}\text { Giraffe, } \\
\text { GAA }\end{array}$ & $\begin{array}{c}\text { Siberian Roe } \\
\text { Deer, CPY }\end{array}$ & $\begin{array}{l}\text { Eurasian } \\
\text { Elk, AAL } \\
\end{array}$ & $\begin{array}{c}\text { Fallow } \\
\text { Deer, DDA }\end{array}$ & $\begin{array}{c}\text { Pere David's } \\
\text { Deer, EDA }\end{array}$ & $\begin{array}{l}\text { Muskox, } \\
\text { OMO }\end{array}$ & $\begin{array}{l}\text { Goat, } \\
\text { CHI }\end{array}$ & $\begin{array}{l}\text { Sheep, } \\
\text { OAR }\end{array}$ & $\begin{array}{c}\text { Sable } \\
\text { Antelope, HNI }\end{array}$ \\
\hline 1 & \multirow{7}{*}{ 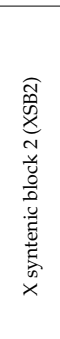 } & CH240- & $\begin{array}{l}\text { Start 1949353, } \\
\text { End 2129088 }\end{array}$ & $66 \mathrm{H} 2$ & $66 \mathrm{H} 2$ & $66 \mathrm{H} 2$ & $66 \mathrm{H} 2$ & $66 \mathrm{H} 2$ & $108 \mathrm{D} 16$ & $386 \mathrm{M} 8$ & $386 \mathrm{M} 8$ & $386 \mathrm{M} 8$ & $93 \mathrm{~K} 24$ & 514022 & $66 \mathrm{H} 2$ & $66 \mathrm{H} 2$ & $66 \mathrm{H} 2$ & $66 \mathrm{H} 2$ \\
\hline 2 & & CH240:- & $\begin{array}{l}\text { Start 7324034, } \\
\text { End 7488466 }\end{array}$ & 155A13 & 155A13 & $155 \mathrm{~A} 13$ & $155 \mathrm{~A} 13$ & 155A13 & $54 \mathrm{D} 24$ & 103Е10 & 103E10 & 103E10 & $122 \mathrm{~N} 13$ & $287 \mathrm{O} 21$ & 155A13 & 155A13 & 155A13 & 155A13 \\
\hline 3 & & CH240- & $\begin{array}{l}\text { Start } 8233624, \\
\text { End } 8391009\end{array}$ & 90L14 & $90 \mathrm{~L} 14$ & 90L14 & 90L14 & 90L14 & $93 \mathrm{~K} 24$ & 22915 & 229115 & 229115 & 195]23 & $128 \mathrm{C} 9$ & 90L14 & 90L14 & $90 \mathrm{~L} 14$ & 90L14 \\
\hline 4 & & CH240- & $\begin{array}{l}\text { Start 13355128, } \\
\text { End } 13540519\end{array}$ & 373L23 & $373 \mathrm{~L} 23$ & $373 \mathrm{~L} 23$ & 373L23 & $373 \mathrm{~L} 23$ & $122 \mathrm{~N} 13$ & $106 \mathrm{~A} 3$ & $106 \mathrm{~A} 3$ & 106A3 & $316 \mathrm{D} 2$ & $106 \mathrm{~A} 3$ & 373L23 & $373 \mathrm{~L} 23$ & $373 \mathrm{~L} 23$ & 373L23 \\
\hline 5 & & CH240:- & $\begin{array}{l}\text { Start 13805346, } \\
\text { End 13950311 }\end{array}$ & $62 \mathrm{M} 10$ & $62 \mathrm{M} 10$ & 62M10 & $62 \mathrm{M} 10$ & 62M10 & $195 / 23$ & $128 \mathrm{C} 9$ & $128 \mathrm{C} 9$ & $128 \mathrm{C} 9$ & $386 \mathrm{M} 8$ & 229115 & 62M10 & $62 \mathrm{M} 10$ & $62 \mathrm{M} 10$ & $62 \mathrm{M} 10$ \\
\hline 6 & & CH240- & $\begin{array}{l}\text { Start 20150516, } \\
\text { End 20286173 }\end{array}$ & $122 \mathrm{P} 17$ & $122 \mathrm{P} 17$ & $122 \mathrm{P} 17$ & $122 \mathrm{P} 17$ & $122 \mathrm{P} 17$ & 316D2 & 287021 & 287021 & $287 \mathrm{O} 21$ & $103 \mathrm{E} 10$ & 103E10 & $122 \mathrm{P} 17$ & $122 \mathrm{P} 17$ & $122 \mathrm{P} 17$ & 122P17 \\
\hline 7 & & CH240:- & $\begin{array}{l}\text { Start 33395588, } \\
\text { End 33587168 }\end{array}$ & $252 \mathrm{G} 15$ & $252 \mathrm{G} 15$ & $252 \mathrm{G} 15$ & $252 \mathrm{G} 15$ & 252G15 & $514 \mathrm{O} 22$ & 514022 & $514 \mathrm{O} 22$ & 514022 & 229115 & $386 \mathrm{M} 8$ & $252 \mathrm{G} 15$ & $252 \mathrm{G} 15$ & $252 \mathrm{G} 15$ & $252 \mathrm{G} 15$ \\
\hline 8 & \multirow{6}{*}{ 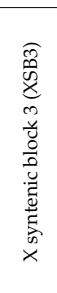 } & CH240- & $\begin{array}{l}\text { Start 48672324, } \\
\text { End } 4817704\end{array}$ & $375 \mathrm{C} 5$ & $375 \mathrm{C} 5$ & $375 \mathrm{C} 5$ & $375 \mathrm{C} 5$ & $375 \mathrm{C} 5$ & 287021 & $316 \mathrm{D} 2$ & $316 \mathrm{D} 2$ & $316 \mathrm{D} 2$ & $106 \mathrm{~A} 3$ & $108 \mathrm{D} 16$ & $375 \mathrm{C} 5$ & $375 \mathrm{C} 5$ & $375 \mathrm{C} 5$ & $375 \mathrm{C} 5$ \\
\hline 9 & & CH240- & $\begin{array}{l}\text { Start 5321958, } \\
\text { End 53351583 }\end{array}$ & 130115 & 130115 & 130115 & 130115 & 130115 & $128 \mathrm{C} 9$ & 1955223 & $195 \sqrt{23}$ & 195523 & 229115 & $54 \mathrm{D} 24$ & 130115 & 130115 & 130115 & 130115 \\
\hline 10 & & $\mathrm{CH}_{240-}$ & $\begin{array}{l}\text { Start 57734547, } \\
\text { End 57947720 }\end{array}$ & 118P13 & $118 \mathrm{P} 13$ & 118P13 & 118P13 & 118P13 & 106A3 & $122 \mathrm{~N} 13$ & $122 \mathrm{~N} 13$ & $122 \mathrm{~N} 13$ & $287 \mathrm{O} 21$ & 93К24 & $118 \mathrm{P} 13$ & $118 \mathrm{P} 13$ & $118 \mathrm{P} 13$ & $118 \mathrm{P} 13$ \\
\hline 11 & & CH240- & $\begin{array}{l}\text { Start 62228039, } \\
\text { End 62371946 }\end{array}$ & $25 \mathrm{P} 8$ & $25 \mathrm{P} 8$ & $25 \mathrm{P} 8$ & $25 \mathrm{P} 8$ & $25 \mathrm{P} 8$ & 229115 & 93К24 & 93К24 & 93К24 & 514022 & $122 \mathrm{~N} 13$ & $25 \mathrm{P} 8$ & $25 \mathrm{P} 8$ & 25P8 & $25 \mathrm{P} 8$ \\
\hline 12 & & CH240- & $\begin{array}{l}\text { Start 62982639, } \\
\text { End } 63183460\end{array}$ & $14 \mathrm{O} 10$ & 14010 & $14 \mathrm{O} 10$ & $14 \mathrm{O} 10$ & $14 \mathrm{O} 10$ & 103E10 & $54 \mathrm{D} 24$ & $54 \mathrm{D} 24$ & $54 \mathrm{D} 24$ & $54 \mathrm{D} 24$ & $195 J 23$ & $14 \mathrm{O} 10$ & $14 \mathrm{O} 10$ & 14010 & $14 \mathrm{O} 10$ \\
\hline 13 & & CH240-: & $\begin{array}{l}\text { Start 68490278, } \\
\text { End } 68678635\end{array}$ & $214 \mathrm{~A} 3$ & $214 \mathrm{~A} 3$ & $214 \mathrm{~A} 3$ & $214 \mathrm{~A} 3$ & $214 \mathrm{~A} 3$ & $386 \mathrm{M} 8$ & $108 \mathrm{D} 16$ & $108 \mathrm{D} 16$ & $108 \mathrm{D} 16$ & $108 \mathrm{D} 16$ & $316 \mathrm{D} 2$ & $214 \mathrm{~A} 3$ & $214 \mathrm{~A} 3$ & $214 \mathrm{~A} 3$ & $214 \mathrm{~A} 3$ \\
\hline 14 & \multirow{13}{*}{ 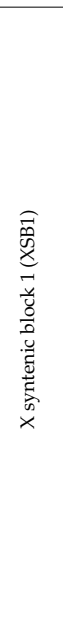 } & CH240:- & $\begin{array}{l}\text { Start 84397606, } \\
\text { End 84521707 }\end{array}$ & $108 \mathrm{D} 16$ & $108 \mathrm{D} 16$ & $108 \mathrm{D} 16$ & $108 \mathrm{D} 16$ & $316 \mathrm{D} 2$ & $214 \mathrm{~A} 2$ & $214 \mathrm{~A} 3$ & $214 \mathrm{~A} 3$ & $214 \mathrm{~A} 3$ & $214 \mathrm{~A} 3$ & $214 \mathrm{~A} 3$ & 386M8 & $386 \mathrm{M} 8$ & $386 \mathrm{M} 8$ & $386 \mathrm{M} 8$ \\
\hline 15 & & CH240- & $\begin{array}{l}\text { Start 85224265, } \\
\text { End 85389684 }\end{array}$ & $54 \mathrm{D} 24$ & $54 \mathrm{D} 24 ?$ & $54 \mathrm{D} 24$ & $54 \mathrm{D} 24$ & $195 \mathrm{~J} 23$ & 1409 & 14010 & $14 \mathrm{O} 10$ & $14 \mathrm{O} 10$ & $14 \mathrm{O} 10$ & $14 \mathrm{O} 10$ & 103E10 & $103 \mathrm{E} 10$ & $103 \mathrm{E} 10$ & 103E10 \\
\hline 16 & & CH240:- & $\begin{array}{l}\text { Start 90681870, } \\
\text { End } 90861947\end{array}$ & 93К 24 & 93К 24 & $93 \mathrm{~K} 24$ & 93К24 & $122 \mathrm{~N} 13$ & 25P7 & 25P8 & 25P8 & 25P8 & 25P8 & 25P8 & $128 \mathrm{C} 9$ & $128 \mathrm{C} 9$ & $128 \mathrm{C} 9$ & 229115 \\
\hline 17 & & CH240- & $\begin{array}{l}\text { Start } 92264186, \\
\text { End } 92429310\end{array}$ & $122 \mathrm{~N} 13$ & $122 \mathrm{~N} 13$ & $122 \mathrm{~N} 13$ & $122 \mathrm{~N} 13$ & 93К 24 & 118P12 & $118 \mathrm{P} 13$ & $118 \mathrm{P} 13$ & $118 \mathrm{P} 13$ & $118 \mathrm{P} 13$ & $118 \mathrm{P} 13$ & $106 \mathrm{~A} 3$ & $106 \mathrm{~A} 3$ & 106A3 & $106 \mathrm{~A} 3$ \\
\hline 18 & & CH240- & $\begin{array}{l}\text { Start 95938488, } \\
\text { End 96135558 }\end{array}$ & 195/23 & $195 \mathrm{~J} 23$ & $195 J 23$ & $195 / 23$ & $54 \mathrm{D} 24$ & 130114 & 130115 & 130115 & 130115 & 130115 & 130115 & 229115 & 229115 & 22915 & $128 \mathrm{C} 9$ \\
\hline 19 & & CH240-: & $\begin{array}{l}\text { Start 103959199, } \\
\text { End 104119579 }\end{array}$ & $316 \mathrm{D} 2$ & $316 \mathrm{D} 2$ & $316 \mathrm{D} 2$ & $316 \mathrm{D} 2$ & 514022 & $375 \mathrm{C} 4$ & $375 \mathrm{C} 5$ & $375 \mathrm{C} 5$ & $375 \mathrm{C} 5$ & $375 \mathrm{C} 5$ & $375 \mathrm{C} 5$ & $287 \mathrm{O} 21$ & 287021 & 287021 & $287 \mathrm{O} 21$ \\
\hline 20 & & CH240:- & $\begin{array}{l}\text { Start 108195394, } \\
\text { End 108349350 }\end{array}$ & $514 \mathrm{O} 22$ & 514022 & $514 \mathrm{O} 22$ & 514022 & 287021 & 252G14 & $252 \mathrm{G} 15$ & 252G15 & $252 \mathrm{G} 15$ & $252 \mathrm{G} 15$ & $252 \mathrm{G} 15$ & $514 \mathrm{O} 22$ & 514022 & 514022 & $514 \mathrm{O} 22$ \\
\hline 21 & & CH240- & $\begin{array}{l}\text { Start 110284444, } \\
\text { End 110450903 }\end{array}$ & 287021 & 287021 & 287021 & 287021 & $128 \mathrm{C} 9$ & $122 \mathrm{P} 16$ & $122 \mathrm{P} 17$ & $122 \mathrm{P} 17$ & $122 P 17$ & $122 \mathrm{P} 17$ & $122 \mathrm{P} 17$ & $316 \mathrm{D} 2$ & $316 \mathrm{D} 2$ & $316 \mathrm{D} 2$ & $316 \mathrm{D} 2$ \\
\hline 22 & & CH240-1 & $\begin{array}{l}\text { Start 111125731, } \\
\text { End 111275450 }\end{array}$ & $128 \mathrm{C} 9 ?$ & $128 \mathrm{C} 9$ & $128 \mathrm{C} 9$ & $128 \mathrm{C} 9 ?$ & $106 \mathrm{~A} 3$ & $62 \mathrm{M} 9$ & $62 \mathrm{M} 10$ & $62 \mathrm{M} 10$ & $62 \mathrm{M} 10$ & $62 \mathrm{M} 10$ & $62 \mathrm{M} 10$ & $195 \sqrt{23}$ & $195 \sqrt{23}$ & $195 / 23$ & $195 \mathrm{~J} 23$ \\
\hline 23 & & CH240:- & $\begin{array}{l}\text { Start 117191008, } \\
\text { End 117311368 }\end{array}$ & $106 \mathrm{~A} 3$ & $106 \mathrm{~A} 3$ & $106 \mathrm{~A} 3$ & $106 \mathrm{~A} 3$ & 229115 & $373 \mathrm{~L} 22$ & 373L23 & $373 \mathrm{~L} 23$ & 373L23 & $373 \mathrm{~L} 23$ & $373 \mathrm{~L} 23$ & $122 \mathrm{~N} 13$ & $122 \mathrm{~N} 13$ & $122 \mathrm{~N} 13$ & $122 \mathrm{~N} 13$ \\
\hline 24 & & CH240-' & $\begin{array}{l}\text { Start 126821940, } \\
\text { End } 127050706\end{array}$ & 229115 & 229115 & 229115 & 229115 & $108 \mathrm{D} 16$ & 90L13 & 90L14 & 90L14 & 90L14 & 90L14 & 90L14 & $93 \mathrm{~K} 24$ & $93 K 24$ & 93К 24 & 93К24 \\
\hline 25 & & CH240- & $\begin{array}{l}\text { Start 128339848, } \\
\text { End 128504608 }\end{array}$ & 103E10 & 103E10 & 103E10 & 103E9 & 103E10 & 155A12 & 155A 13 & $155 \mathrm{~A} 13$ & $155 \mathrm{~A} 13$ & $155 \mathrm{~A} 13$ & 155A13 & $54 \mathrm{D} 24$ & $54 \mathrm{D} 24$ & $54 \mathrm{D} 24$ & $54 \mathrm{D} 24$ \\
\hline 26 & & CH240-- & $\begin{array}{l}\text { Start 141101222, } \\
\text { End 141358968 }\end{array}$ & $386 \mathrm{M} 8$ & $386 \mathrm{M} 8$ & $386 \mathrm{M} 8$ & $386 \mathrm{M} 7$ & $386 \mathrm{M} 8$ & $66 \mathrm{H} 1$ & $66 \mathrm{H} 2$ & $66 \mathrm{H} 2$ & $66 \mathrm{H} 2$ & $66 \mathrm{H} 2$ & $66 \mathrm{H} 2$ & $108 \mathrm{D} 16$ & $108 \mathrm{D} 16$ & $108 \mathrm{D} 16$ & 108D16 \\
\hline
\end{tabular}




\section{Result}

\subsection{BACs Localization}

We investigated the $X$ chromosome structure across major branches of Cetartiodactyla represented by 18 species from four non-ruminant (Suidae, Camelidae, Eschrichtiidae (Cetacea), Hippopotamidae) and six ruminant (Tragulidae, Antilocapridae, Giraffidae, Moschidae, Cervidae, and Bovidae) families (Table 1). The order of 26 labeled cattle BAC clones was established on the X chromosomes of each of 18 species by a series of pairwise FISH experiments (Table 2). In total, comparative analyses of BAC orders across 18 species revealed three major chromosomal conservative segments, which were numbered and designated with colors used throughout the paper: X Syntenic Block 1 (13 BACs, XSB1, pink); X Syntenic Block 2 (seven BACs, XSB2, yellow), and; X Syntenic Block 3 (six BACs, XSB3, blue).

\subsection{Intrachromosome Rearrangements}

Comparative analysis of the order of BAC on the $\mathrm{X}$ chromosomes of 18 species identified three key scenarios that likely took place in the course of the cetartiodactyl X chromosomes' evolution.

1. Conservation: no change in the BAC order and no change of the centromere position. We identified a group of four basal cetartiodactyl species (gray whale (ERO), common hippopotamus (HAM), alpaca (LPA), and pig (SSC)) that have an identical order of the BACs and the same relative position of the centromere (located in XSB1).

2. Centromere repositioning: conserved BAC order, changes in the centromere position. Centromere repositions have been shown in roe deer, and mouse-deer, resulting in metacentric (Siberian roe deer (CPY)) and acrocentric (Java mouse-deer (TJA)) X chromosomes, respectively. This event took place prior to a formation of some lineage specific ancestral chromosomes (RAX (Ruminant Ancestral X), AAX ( Antilopinae Ancestral X), and CEAX (Cervinae Ancestral X)), indicating that centromere repositioning is one of the key rearrangements of the ruminant $X$ : while maintaining a conserved order of the segments there was a displacement of the centromere (Figure 1).

3. Inversion: changes in the BAC order. Three kinds of inversions were identified: (A) syntenic block (SB) flip — this inversion reverses the orientation of the whole syntenic block (TJA, AAM, $\mathrm{AAX}, \mathrm{CEAX}$ ); (B) an inversion inside the syntenic block (goat (CHI), muskox (OMO)); (C) the exchange inversion-inversion that involves several BAC clones from two syntenic blocks (TJA, fallow deer (DDA)) (Figure 2).

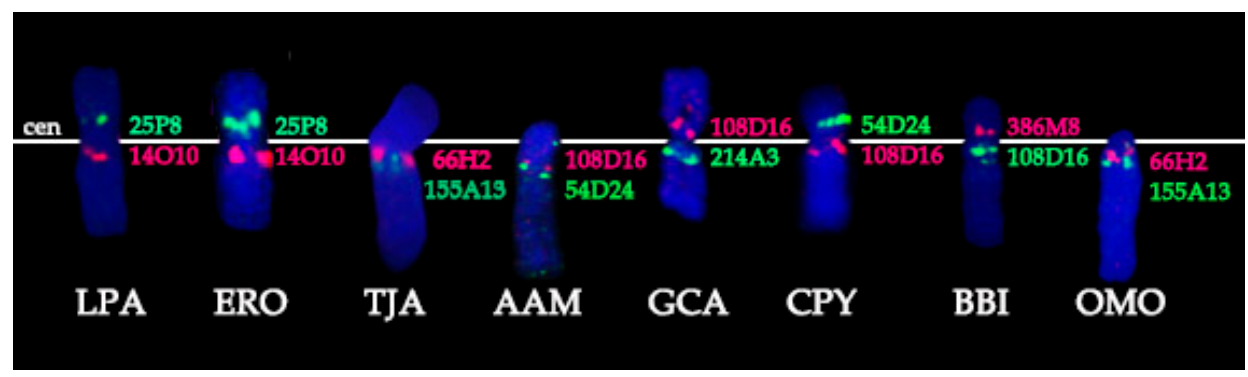

Figure 1. Centromere location (cen, white line) and positions of specific BAC clones (pink and green) on X chromosome of several cetartiodactyl species. Species three-letter codes are listed in Table 1.

Taken together, we found that inversions (paracentric and pericentric) and centromere shifts were key rearrangements in the course of $X$ chromosome evolution in Cetartiodactyla. In addition to the described rearrangements, the nucleolar organizing region (containing clusters of $18 \mathrm{~S}$ and 28S rDNA genes) were localized on the short arm of both $X$ and $Y$ sex chromosomes of the Java mouse-deer (TJA) [33]. 


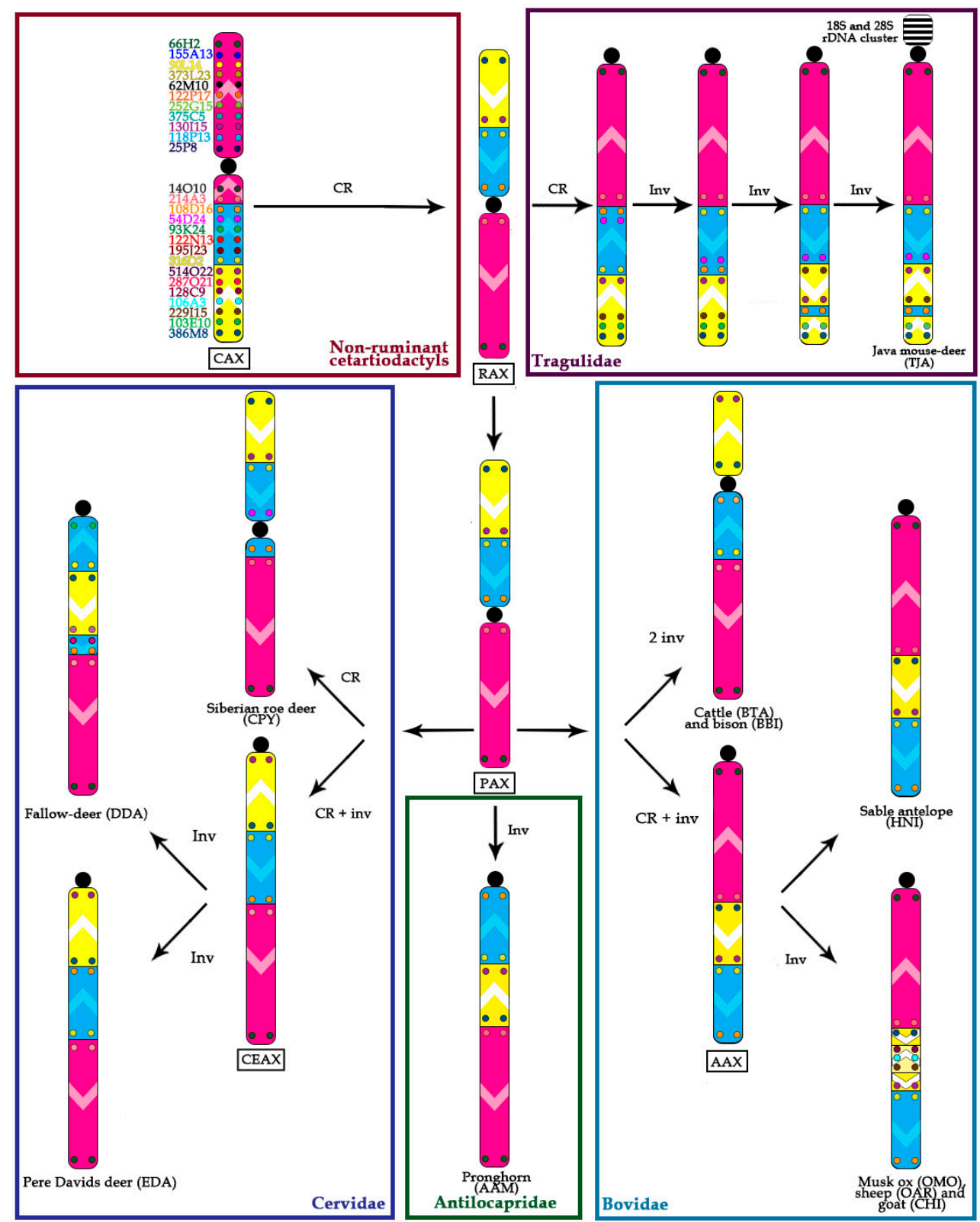

Figure 2. The scheme of evolutionary transformations of $\mathrm{X}$ chromosome in Cetartiodactyla. Chromosome rearrangements were identified by changes in BAC order. Three major conservative segments are designated by different colors: pink-X syntenic block 1 ; yellow-X syntenic block 2 , and; blue-X syntenic block 3 . Individual BAC clones are shown with a different color in small colored circles on corresponding conservative segment. Centromere position is indicated by a black circle. The orientation of the conservative segments is indicated by the white arrowhead. Ancestral associations are shown in black rectangle (Cetartiodactyla ancestral X (CAX), Ruminantia ancestral $X(R A X)$, Pecora ancestral X (PAX), Antilopinae ancestral X (AAX), Cervinae ancestral X (CEAX)). $\mathrm{CR}$ : centromere reposition. Inv: inversion. 
Table 3. CHORI-240 (CH-240) BAC's order on mammalian chromosomes X. Conservative syntenic segments are colored in pink, yellow and blue.

\begin{tabular}{|c|c|c|c|c|c|c|c|c|c|c|c|c|}
\hline \multirow[b]{2}{*}{ No. } & \multicolumn{6}{|c|}{ Laurasiatheria } & \multicolumn{6}{|c|}{ Euarchontoglires } \\
\hline & \multicolumn{2}{|c|}{$\begin{array}{c}\text { BAC Clones in Cattle } \\
\text { Genome }\end{array}$} & \multicolumn{2}{|c|}{$\begin{array}{c}\text { BAC Clones in Sheep } \\
\text { Genome }\end{array}$} & \multicolumn{2}{|c|}{$\begin{array}{c}\text { BAC Clones in Horse } \\
\text { Genome) }\end{array}$} & \multicolumn{2}{|c|}{$\begin{array}{c}\text { BAC Clones in Human } \\
\text { Genome }\end{array}$} & \multicolumn{2}{|c|}{$\begin{array}{l}\text { BAC Clones in Mouse } \\
\text { Genome }\end{array}$} & \multicolumn{2}{|c|}{ BAC Clones in Rat Genome } \\
\hline 1 & 514022 & $\begin{array}{l}\text { Start } 1949353 \\
\text { End } 2129088\end{array}$ & $66 \mathrm{H} 2$ & $\begin{array}{l}\text { Start } 10045822 \\
\text { End } 10306770\end{array}$ & $66 \mathrm{H} 2$ & $\begin{array}{l}\text { Start } 8367618 \\
\text { End } 8624882\end{array}$ & $66 \mathrm{H} 2$ & $\begin{array}{l}\text { Start } 12497685 \\
\text { End } 12794877\end{array}$ & 118P13 & $\begin{array}{l}\text { Start 7554450 } \\
\text { End 7697987 }\end{array}$ & $25 \mathrm{P} 8$ & $\begin{array}{l}\text { Start } 1711095 \\
\text { End } 1907049\end{array}$ \\
\hline & & Start 7324034 & & Start 19299853 & & Start 16543677 & & Start 22069138 & & Start 9209615 & & Start 4672236 \\
\hline 2 & 287021 & End 7488466 & 155A13 & End 19464920 & 155A13 & End 16698622 & 155A13 & End 22228453 & 62M10 & End 9317028 & $375 C$ & End 4863802 \\
\hline & & Start 8233624 & & Start 28630482 & & Start 24698243 & & Start 31328065 & & Start 10195810 & & Start 10936630 \\
\hline 3 & $128 \mathrm{C} 9$ & End 8391009 & 373L23 & End 28810179 & 373L23 & End 24857300 & $373 \mathrm{~L} 23$ & End 3150 & $122 \mathrm{P} 17$ & nd 103 & 252G15 & End 11107682 \\
\hline 4 & & Start 13345128 & $69 \mathrm{M}$ & Start 34891275 & $6 ?$ & Start 30220096 & Mi & Start 31328065 & 15 & art 126 & $122 \mathrm{P} 17$ & 183272 \\
\hline 4 & $106 \mathrm{A3}$ & End 13540519 & $62 \mathrm{M}$ & End 35 & $62 \mathrm{M}$ & End 30 & 2M10 & End 315 & $252 \mathrm{G} 15$ & 54 & $122 P 17$ & 671 \\
\hline 5 & 229115 & $\begin{array}{l}\text { Start } 13805346 \\
\text { Fnd } 13950311\end{array}$ & $122 \mathrm{P} 17$ & $\begin{array}{l}\text { Start } 35738657 \\
\text { End } 35910824\end{array}$ & $122 \mathrm{P} 17$ & Start 30907267 & $122 \mathrm{P} 17$ & $\begin{array}{l}\text { Start } 38298814 \\
\text { End } 38458494\end{array}$ & $375 \mathrm{C} 5$ & Fnd 1848 & 62M10 & 15064 \\
\hline & & Start 20150516 & & Start 37830134 & & Start 32879937 & & $\begin{array}{l}\text { End } 384584494 \\
\text { Start } 40611820\end{array}$ & & Start 20507324 & & $\begin{array}{l}\text { End } 14451123 \\
\text { Start } 15650399\end{array}$ \\
\hline 6 & 103E1 & End 20286173 & 252G15 & End 37981845 & $252 \mathrm{G} 15$ & End 33007527 & 252G15 & End 40767797 & 25P8 & nd 20696050 & $118 \mathrm{P} 13$ & End 15784402 \\
\hline 7 & 386M8 & $\begin{array}{l}\text { Start } 33395588 \\
\text { End } 33587168\end{array}$ & $375 \mathrm{C} 5$ & $\begin{array}{l}\text { Start } 41973255 \\
\text { End } 42128838\end{array}$ & $375 \mathrm{C} 5$ & $\begin{array}{c}\text { Start } 36512266 \\
\text { End } 36698919\end{array}$ & $375 \mathrm{C} 5$ & $\begin{array}{l}\text { Start } 45036869 \\
\text { End } 45234319\end{array}$ & $514 \mathrm{O} 22$ & $\begin{array}{l}\text { Start } 23213727 \\
\text { End } 2331629\end{array}$ & 130115 & $\begin{array}{l}\text { Start } 22235385 \\
\text { End } 22435973\end{array}$ \\
\hline 8 & $108 \mathrm{D} 16$ & $\begin{array}{l}\text { Start } 48672324 \\
\text { End } 48917704\end{array}$ & 130115 & $\begin{array}{l}\text { Start } 49649383 \\
\text { End } 49847996\end{array}$ & 8 & $\begin{array}{l}\text { Start } 38190847 \\
\text { End } 38327897\end{array}$ & P8 & $\begin{array}{l}\text { Start } 47047149 \\
\text { Fnd } 47226311\end{array}$ & 287021 & $\begin{array}{r}\text { Start } 41535889 \\
\text { Fnd } 4167049\end{array}$ & $66 \mathrm{H} 2$ & $\begin{array}{l}\text { Start } 27957571 \\
\text { End } 28439731\end{array}$ \\
\hline 9 & $54 \mathrm{D} 24$ & $\begin{array}{l}\text { Start } 53219586 \\
\end{array}$ & $118 \mathrm{P}$ & $\begin{array}{l}\text { Start } 52564228 \\
\end{array}$ & 11 & $\begin{array}{l}\text { Start } 39580949 \\
\text { End } 39734268\end{array}$ & & $\begin{array}{l}\text { Srart } 49122932 \\
\text { End } 49608099\end{array}$ & 59 & 010 & 155A13 & \\
\hline 9 & & & 110110 & & & & $8 \mathrm{PP}$ & & -9 & & 100A15 & End \\
\hline 10 & $93 \mathrm{~K} 2$ & $\begin{array}{l}\text { Start } 57734547 \\
\text { End } 57947720\end{array}$ & $25 \mathrm{P} 8$ & $\begin{array}{l}\text { Start } 54170178 \\
\text { End 54331345 }\end{array}$ & 130115 & $\begin{array}{l}\text { Start } 44962739 \\
\text { End } 45135718\end{array}$ & 130115 & $\begin{array}{l}\text { Start } 53053920 \\
\text { End } 53291737\end{array}$ & $106 \mathrm{~A} 3$ & $\begin{array}{l}\text { Start } 4 \\
\text { End } 4\end{array}$ & $373 \mathrm{~L} 23$ & $\begin{array}{l}\text { Start } 53052665 \\
\text { End } 53277814\end{array}$ \\
\hline & & Start 62228039 & & Start 59810734 & & $\begin{array}{l}\text { nna } 4531301818 \\
\text { Start } 52316685\end{array}$ & & Start 70333575 & & & & 930 \\
\hline 11 & $122 \mathrm{~N} 13$ & End 62371946 & 1401 & & $14 \mathrm{O}$ & & 14010 & & 229115 & & $14 \mathrm{O}$ & \\
\hline 12 & $195 J 23$ & $\begin{array}{l}\text { Start } 62982639 \\
\text { End } 63183460\end{array}$ & 214A3 & $\begin{array}{l}\text { Start } 60702841 \\
\text { End } 60821565\end{array}$ & $214 \mathrm{~A} 3$ & $\begin{array}{l}\text { Start } 53269385 \\
\text { Fnd } 53289760\end{array}$ & $214 \mathrm{~A} 3$ & $\begin{array}{l}\text { Start } 71438703 \\
\text { Fnd } 71567090\end{array}$ & 103E10 & $\begin{array}{l}\text { Start } 57106307 \\
\text { End } 57244888\end{array}$ & $214 \mathrm{~A} 3$ & 8323 \\
\hline & & $\begin{array}{l}\text { End } 631834600 \\
\text { Start } 68490278\end{array}$ & & $\begin{array}{l}\text { End } 680211565 \\
\text { Start } 80094458\end{array}$ & & $\begin{array}{l}\text { End } 533399760 \\
\text { Start } 76549898\end{array}$ & & $\begin{array}{l}\text { End } 71567 \\
\text { Start } 97540\end{array}$ & & $\begin{array}{l}\text { End } 572 \\
\text { Start 711 }\end{array}$ & & \\
\hline 13 & $316 \mathrm{D} 2$ & End 68678635 & $386 \mathrm{M} 8$ & End & $108 \mathrm{D} 16$ & & $108 D 16$ & & $386 \mathrm{M} 8$ & End 71 & $108 \mathrm{D} 16$ & 62 \\
\hline 14 & $214 \mathrm{~A}$ & $\begin{array}{l}\text { Start } 843 \\
\text { End } 8452\end{array}$ & $103 \mathrm{E}$ & $\begin{array}{l}\text { Start } 93391997 \\
\text { End } 93531761\end{array}$ & $93 \mathrm{~K} 24$ & $\begin{array}{l}\text { Start } 817 \\
\text { End } 818\end{array}$ & $54 \mathrm{D} 24$ & $\begin{array}{l}\text { Start } 103662230 \\
\text { End } 103838933\end{array}$ & 23 & $\begin{array}{l}\text { Start } 847 \\
\text { End } 849\end{array}$ & 93K2 & $\begin{array}{l}8470 \\
2526\end{array}$ \\
\hline & & & & & & & & & & & & \\
\hline 15 & $14 \mathrm{O} 10$ & $\begin{array}{l}\text { Start } 85 \\
\text { End } 85\end{array}$ & $287 \mathrm{O} 21$ & $\begin{array}{l}\text { Start 10 } \\
\text { End } 10\end{array}$ & $54 \mathrm{D} 24$ & $\begin{array}{l}\text { Start } \\
\text { End }\end{array}$ & 93K24 & 58 & $14 \mathrm{O} 10$ & $\begin{array}{l}\text { tart } \\
\text { End }\end{array}$ & $54 \mathrm{D} 24$ & End \\
\hline 16 & 25P8 & Start 90681870 & $128 \mathrm{C} 9$ & $\begin{array}{l}\text { Start } 10 \\
\text { End } 10\end{array}$ & $122 \mathrm{~N} 13$ & Start 86 & $122 \mathrm{~N} 13$ & Start 109 & $214 \mathrm{~A} 3$ & Start 1 & $122 \mathrm{~N} 13$ & Start 1 \\
\hline & & & & & & & $122 \mathrm{~N} 13$ & & & & 122N13 & \\
\hline 17 & $118 \mathrm{P} 13$ & $\begin{array}{l}\text { Start } 92264186 \\
\text { End } 92429310\end{array}$ & $106 \mathrm{~A} 3$ & $\begin{array}{l}\text { Start } 107701336 \\
\text { End } 107885819\end{array}$ & 195J23 & $\begin{array}{l}\text { Start } 86 \\
\text { End } 87\end{array}$ & $195 J 23$ & Start 11 & 108D16 & $\begin{array}{l}\text { Start } 1 \\
\text { Fnd } 1 \text { ? }\end{array}$ & $195 \mathrm{~J} 23$ & 201 \\
\hline & & Start 95 & & Start 10815 & & $\begin{array}{l}\text { nend } 8 \\
\text { Start } 8\end{array}$ & & $\begin{array}{l}\text { Enc } \\
\text { Star }\end{array}$ & & $\begin{array}{l}\text { En } \\
\text { Sta }\end{array}$ & & \\
\hline 18 & 13011 & & I15 & & $316 \mathrm{D} 2$ & & 16D2 & & K24 & & 316D2 & \\
\hline 19 & $375 \mathrm{C}$ & $\begin{array}{l}\text { Start } 103959199 \\
\text { End } 104119579\end{array}$ & $514 \mathrm{O} 22$ & $\begin{array}{l}\text { Start } 111218064 \\
\text { Fnd } 111402064\end{array}$ & $514 \mathrm{O} 22$ & $\begin{array}{l}\text { Start } 93641183 \\
\text { Fnd } 9380197\end{array}$ & $40 \angle 2$ & $\begin{array}{l}\text { Start 1178847474 } \\
\text { Fnd } 118065524\end{array}$ & 4D24 & $\begin{array}{l}\text { Start } 138 \\
\text { End } 138\end{array}$ & & 459 \\
\hline & & & & $\begin{array}{l}\text { End } \\
\text { Start }\end{array}$ & & $\begin{array}{l}\text { End } \\
\text { Star }\end{array}$ & & & & & 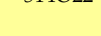 & \\
\hline 20 & 252G15 & End 108 & $316 \mathrm{D} 2$ & End 1 & 287021 & End 980 & $287 \mathrm{O} 21$ & End 123. & $122 \mathrm{~N} 13$ & & $287 \mathrm{O} 21$ & 918 \\
\hline 1 & 120P & Start & 19512 & & 11 & Star & 1280 & & 195123 & & 128 & \\
\hline 21 & 12 & & 19552 & & & & & & 19 & & C9 & \\
\hline 22 & $62 \mathrm{M} 10$ & Star & $122 \mathrm{~N} 13$ & Star & $106 \mathrm{~A} 3$ & & $106 \mathrm{~A} 3$ & & $316 \mathrm{D} 2$ & & $106 \mathrm{~A} 3$ & \\
\hline & & & & & & & & & & & & \\
\hline 23 & 373L23 & Start 1 & 93К 24 & Start 122 & 229115 & $\begin{array}{l}\text { Start 1c } \\
\end{array}$ & 229115 & Start 129 & 30115 & 08 & 229115 & Start 1 \\
\hline & & $\begin{array}{l}\text { End } 11 \\
\text { Start } 12\end{array}$ & & Start 124 & & Start 1086 & & Start 13 & & Start & & $\begin{array}{l}\text { End } 1328818018 \\
\text { Start } 1595803\end{array}$ \\
\hline 24 & 155A13 & End 128504608 & $54 \mathrm{D} 24$ & End 124 & 103Е10 & End 108 & 103E10 & End 1366 & 155A13 & End 1573 & 103E10 & End 159734497 \\
\hline 25 & $66 \mathrm{H} 2$ & $\begin{array}{l}\text { Start } 141101222 \\
\text { End } 141358968\end{array}$ & 108D16 & $\begin{array}{l}\text { Start 129843594 } \\
\text { End } 130091258\end{array}$ & $386 \mathrm{M} 8$ & $\begin{array}{l}\text { Start } 119476270 \\
\text { End } 119683931\end{array}$ & 386M8 & $\begin{array}{l}\text { Start } 150502313 \\
\text { End } 150728735\end{array}$ & $66 \mathrm{H} 2$ & $\begin{array}{l}\text { Start } 167378561 \\
\text { End } 167730488\end{array}$ & & \\
\hline
\end{tabular}




\subsection{Bioinformatic Analysis of Mammalian X chromosomes}

To evaluate the unique conservation of mammalian $X$ chromosomes [3] we calculated the coordinates of 26 BAC clone sequences in four Boreoeutherian non-cetartiodactyl genomes represented by Euarchontoglires: human (Primates); mouse, and rat (Rodentia), and; Laurasiatheria: horse (Perissodactyla). We have observed that three X chromosome syntenic blocks (XSB) found in Cetartiodactyla are conserved in Laurasiatheria and also in Euarchontoglires, indicating common Boreoeutherian structure of the $\mathrm{X}$ chromosome. It was previously reported that human, horse, and pig $X$ chromosomes have similar gene order [3]. In general, this observation was confirmed by liftOver analyses (Table 3). We have identified several small inversions in XSB1 (human, horse) and in XSB2 (horse) in comparison to CAX. Interestingly, XSB1 is the most derived segment outside of Cetartiodactyla, no rearrangements in BACs order in the cetartiodactyl species were detected within this block. According to our data, XSB2 is highly conserved in non-cetartiodactyl species, while in ruminants there are inversions inside of this syntenic block (CHI, OMO, sheep (OAR)) and exchange inversions between XSB2 and XSB3 (TJA and DDA).

We also aligned the BAC clone sequences to another cetartiodactyl genome, the domestic sheep. We observed the same BAC order as in all analyzed Caprini species except for a small inversion in XSB3. The FISH with relevant BAC clones confirmed the presence of this inversion in the sheep genome.

\section{Discussion}

\subsection{Ancestral X chromosome}

The phenomenon of $\mathrm{X}$ chromosome conservation in eutherian mammals was first proposed by Susumu Ohno and was based solely on its size similarity across a wide range of species [1]. High similarity in G-banding pattern led to the hypothesis that not only size and gene content [34] but also gene order is conserved on the $\mathrm{X}$ chromosomes of most eutherian mammals, and this was later confirmed by fine gene mapping [3,35-38]. Remarkably, the submetacentric $X$ chromosome morphology defined by the location of the centromere is also largely conserved across mammals. Some slight changes of otherwise conserved $X$ chromosomes were observed in several orders, such as the difference in the distance between homologous genes between human and alpaca [39], or a shift in centromere position without a change of the gene order in Afrotheria [37]. Still, the lack or low level of rearrangements of the $\mathrm{X}$ chromosome in comparison to the active exchanges on autosomes during over 150 million years of eutherian evolution represents an interesting phenomenon. Comparative G-banding analysis had identified the classical chromosome $\mathrm{X}$ morphology and banding pattern common to most eutherian species [2]. Similar submetacentric morphology and gene order were also found in non-ruminant cetartiodactyls. A high level of $\mathrm{X}$ chromosome conservation was shown in Suinae [3,40], Tylopoda [41-43], and Cetacea [44]. Nevertheless, using G-banding analysis [4] and high-resolution mapping with BACs [25] or region specific probes, [12] intrachromosomal rearrangements were uncovered in Ruminantia species. Compared with the previous study, we have expanded the number of BACs to 26 and the species list to 18 in order to define conservative blocks and their orientation, to identify rearrangements across species, and to reconstruct the ancestral cetartiodactyl X chromosome. The analyses of BAC order across major families of Cetartiodactyla revealed three syntenic blocks on the $X$ chromosome that in general correspond to the conserved segments reported by Fröhlich and coauthors [25].

Using available FISH and bioinformatic data on the order of cattle BACs in the genomes of different species, we were able to investigate the phenomenon of the conservation of the $\mathrm{X}$ chromosome in eutherian mammals represented by four superorders: Laurasiatheria; Euarchontoglires; Afrotheria, and; Xenarthra [45]. Three conserved syntenic blocks identified here can be traced in Boreoeutherians (Laurasiatheria and Euarchontoglires), and possibly in all eutherians, considering reports on Afrotheria $X$ chromosome conserved gene order [37] (Table 2). The eutherian $X$ chromosome ancestral condition (EUX) is represented by a submetacentric chromosome with the centromere located in 
XSB1. Bioinformatic analysis in outgroup species shows a common change of BAC order in XSB1 on human and horse X chromosomes. Supposedly, an inversion on EUX had occurred in the ancestor of Cetartiodactyla prior the radiation of this order. This ancestral condition was revealed in all non-ruminant cetartiodactyls and named here Cetartiodactyla Ancestral X (CAX). We confirmed the conservation of the $\mathrm{X}$ chromosome in basal branches of Cetartiodactyla. It occurs in Suidae (pig), Camelidae (alpaca), and Cetacea (gray whale) (Table 2 and Figure 3). Cetacea is a sister taxon to Hippopatamidae and is characterized by extremely conserved karyotypes across the whole infraorder and by uniform $X$ chromosome morphology and banding pattern $[11,46]$. The Hippopotamidae $X$ chromosome also displays the same morphology and the gene order $[8,25]$. However, it should be emphasized that there are some unresolved cases of the $X$ chromosome changes in these basal groups that would require additional investigation, for example, the $X$ chromosome of Tayassu pecari (Suinae, Taysuidae) has been changed due to a centromere reposition [40].

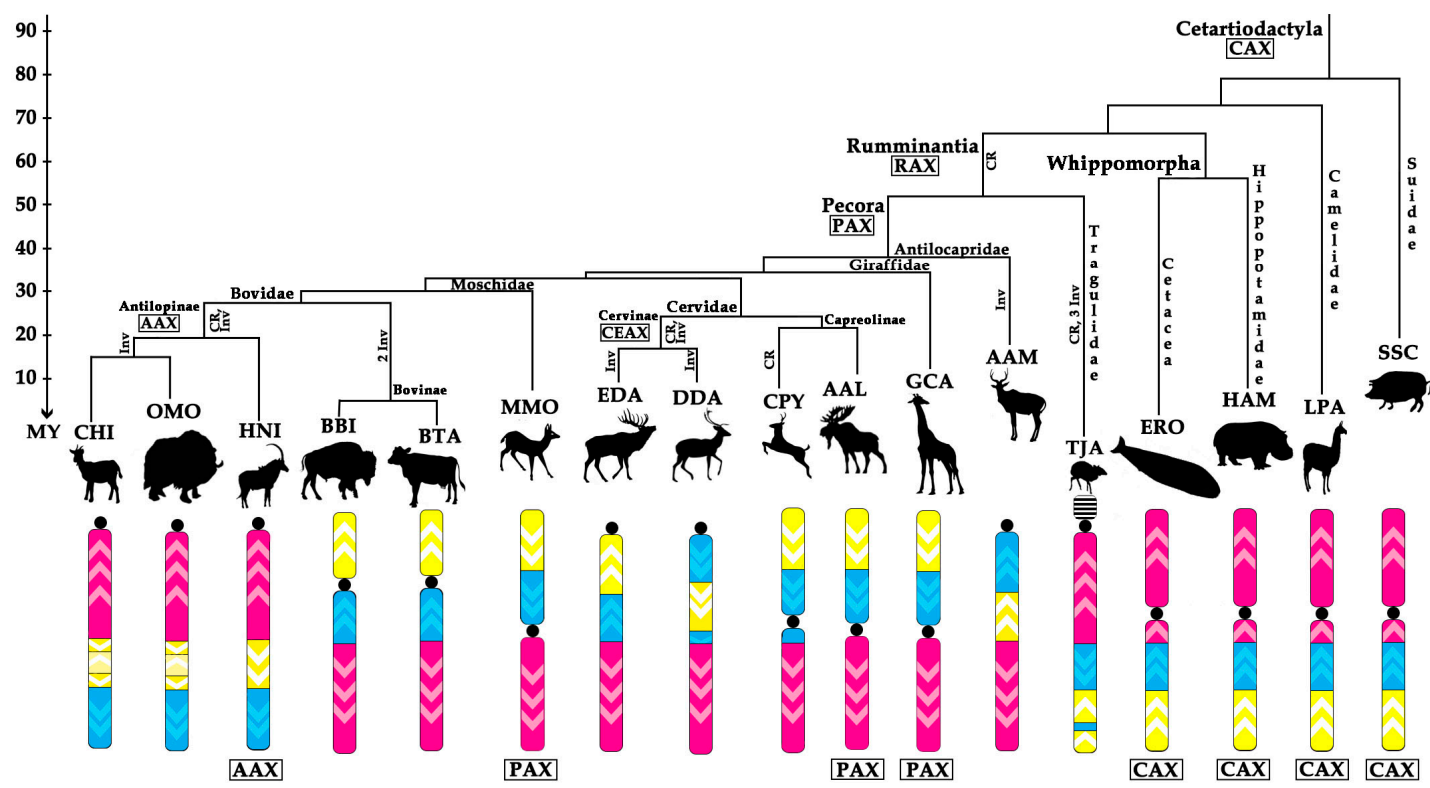

Figure 3. The structure of the Cetartiodactyla $X$ chromosome depicted on the phylogenetic tree of the order (the tree topology from [47]) Major conservative segments are shown by yellow, blue, and pink. Centromere positions are designated by a black circle. White arrowheads show the orientation of the conservative segments. Ancestral associations are shown under X chromosomes (Cetartiodactyla ancestral X (CAX), Ruminantia ancestral X (RAX), Pecora ancestral X (PAX), Antilopinae ancestral X (AAX). MMO X chromosome is inverted here relatively to its cytogenetic orientation for presentation purposes [8].

\subsection{Ancestral Form of Ruminantia-Pecora X-Chromosome}

Contrary to the conservation of the $\mathrm{X}$ chromosome in Suidae-Camelidae-Whippomorpha, we found that multiple rearrangements occurred during the radiation of other cetartiodactyl branches. We suggest that in the Ruminantia an ancestral centromere reposition led to changes of the $\mathrm{X}$ chromosome morphology from submetacentric to metacentric forming the Ruminantia Ancestral X-chromosome (RAX) (Figures 2 and 3). Both ancestral forms (CAX and RAX) have same intrachromosomal structure and differ only by centromere position. The RAX form of the $X$ chromosome is also preserved in many basal Pecora branches: Giraffidae (GCA); Moschidae (MMO); and in the Capreolini (AAL) subfamily of Cervidae. Only in the basal Pecoran family Antilocapridae, an inversion turned the ancestral metacentric $X$ chromosome into an acrocentric element (Figure 2). Thus we expect that the Ancestral Ruminant and the Ancestral Pecoran X chromosomes have the same structure: RAX=PAX. 
In the Tragulidae, the basal and the only non-Pecora ruminant group, we found a major centromere reposition resulted in the formation of an acrocentric X. Also, two kinds of inversions (SB-flip and synteny block exchange) affect syntenic block structure in the Tragulidae. These rearrangements create unique arrangement of the three syntenic blocks in the Java mouse-deer. This arrangement may occur across all tragulids, but requires confirmation in other Tragulus species.

\subsection{Cervidae}

There is a great variation in $\mathrm{X}$ chromosome morphology among cervids. Two cervid subfamilies, Capreolinae and Cervinae, exhibit a notably differing extent of sex chromosome conservation. The only detected rearrangement was a centromere shift in CPY. G-banding pattern comparison of Capreolinae $\mathrm{X}$ chromosomes otherwise indicates a uniform metacentric morphology [48] and suggests a similar disposition of conservative syntenic blocks.

In contrast, Cervinae is characterized by a variety of rearrangements on the $\mathrm{X}$ chromosome: centromere repositioning, SB flips, and many inversions disrupting the XSB2. The Cervinae Ancestral X-chromosome (CEAX) was formed by a centromere reposition and a SB flip of XSB2. Inversions change this ancestral form in EDA by SB flip of XSB3 and in DDA by the splitting of XSB2 (Figure 2). Also in the same subfamily, a translocation of an autosome to the $X$ chromosome was reported in several Muntiacini species [7,49-52]. In total, this indicates that the level of $X$ chromosome variation is increased in Cervinae and is caused not only by inversions and centromere repositioning but also by autosome to sex chromosome translocations.

\subsection{Bovidae}

The family Bovidae includes two major branches: Bovinae and Antilopinae [53]. Earlier cytogenetic studies identified two types of morphological diversity of $\mathrm{X}$ chromosome in Bovidae: a caprine type (acrocentric, suni type) and a bovine type (submetacentric) [12,54]. The bovine type of $\mathrm{X}$ chromosome was likely formed from the ancestral pecoran $\mathrm{X}$ (PAX) by two inversions. This form is retained in cattle (BTA) and American bison (BBI). Cytogenetic data for other studied Bovinae species demonstrated same submetacentric $X$ chromosome morphology [48]. There are independent autosome translocations in two branches (Tragelaphini and Bosephalini) altering the bovine type $\mathrm{X}$ chromosome $[12,23,48,55,56]$. The notable exceptions are the Bubalina lineage, oryx and kudu (Tragelaphilini), whose $X$ chromosomes have acrocentric morphology (designated as eland-type acrocentric based on eland, kudu, and nyala $X$ chromosomes [12]).

Centromere reposition and inversion events resulted in the formation of an acrocentric Antilopinae Ancestral X-chromosome (AAX) (Figure 2) from PAX. Therefore the $X$ of the sable antelope (HNI) could likely represent an ancestral form for all Antilopinae. Moreover, comparative analyses based on published karyotypes supports the theory that the $\mathrm{X}$ chromosome in antelopes is largely conserved, retaining the same morphology and banding pattern [48,57]. The exceptions are autosome to $X$ chromosome translocations found in several Antilopini species $[48,58]$. In the Caprini lineage there is an additional inversion within the XSB3 (OMO, CHI, OAR). The bioinformatic and FISH analyses of X chromosome of OAR indicated that the inversion between 128C9 and 229115 is an apomorphic phylogenetic marker for Caprini.

\subsection{X chromosome Rearrangements}

All $X$ chromosome rearrangements discovered here are in agreement with the current phylogenetic tree (Figure 3), and some of them could be used as cytogenetic markers for different Cetartiodactyla groups. Therefore, we suggest that our BAC clone set can serve as a precise instrument for a further search for cytogenetic $X$ chromosome markers in Bovidae. The independent autosome to sex chromosome translocations that occurred in several Bovidae and Cervidae branches require special attention because they increase the previously identified rapid rate of evolution of the structure of the cetartiodactyl X chromosome $[7,12,49-52,55,56]$. 
The BAC clones that mark the borders of three conserved segments delineate regions of frequent chromosome rearrangements in cetartiodactyl X, indicating a breakpoint reuse phenomenon [59]. Several BAC clones were involved at least twice in the intrachromosomal rearrangements found here, suggesting breakpoint reuse: 108D16 and 214A3; 514O22 and 316D2; $229 \mathrm{I} 15$ and 103E10. We found that the regions surroundings these BACs in the cattle genome are often gene sparse. It was previously shown that chromosomal regions with evolutionary breakpoint in amniotes are enriched for structural variations (segmental duplications, copy number variants, and indels), retrotransposons, zinc finger genes, and single nucleotide polymorphisms [60]. Further investigation is required to find precise points of evolutionary chromosome breakage on the Cetatiodactyla $\mathrm{X}$ and to define common genomic features underlying chromosome rearrangements.

Another mammalian order characterized by the increased rate of $X$ chromosome evolution is Rodentia. Heterochromatin expansion, amplification of tandem repeats, inversions [61], centromere reposition [62], and autosome to sex chromosome translocations [63] were shown to be involved in rearrangements of $\mathrm{X}$ chromosome in rodents. Comparative chromosomal analysis of $\mathrm{X}$ chromosomes was performed by microdissection in the Microtus genus. Rubtsov with coauthors postulated that intrachromosomal rearrangements are associated with large clusters of intrachromosomal duplications and/or repeated DNA sequences which were present in ancestral species but have subsequently disappeared during evolution [61]. We hypothesize that similar processes were involved in evolution of X chromosome in Ruminantia. Some genomic events possibly took place in the ruminant ancestor that launched multiple chromosomal rearrangements of the conservative eutherian $\mathrm{X}$ chromosome. Insertions of mobile repetitive elements such as long and short interspersed nuclear elements (LINE and SINE were probably involved in synteny breaks on this sex chromosome [64]. It is possible that this transforming genomic event had happend in or around the XSB2 area which demonstrates highest rate of inversions in Ruminantia.

In total, nine paracentric, two pericentric inversions, and five centromere reposition events have been revealed in Cetartyodactyla $\mathrm{X}$ chromosome evolution based on the analysis of 18 species. The eutherian and cetartiodactyl ancestral $X$ differ only by one small inversion; one additional rearrangement is proposed to derive the Ruminantia ancestral X (RAX). Most other identified rearrangements happend during the remaining 55 million years of ruminant's radiation. The cow $X$ chromosome was formed by at least two rearrangements that distinguish it from PAX, corresponding to a rate of rearrangements of approximately 1 per 15 million years. This is comparable to 1 rearrangement per 10 million years postulated for autosomal evolution among most mammalian orders found by chromosome painting [65]. These findings are consistent with the rate of $X$ chromosome evolution in Ruminantia being at least twice as high as in $\mathrm{X}$ chromosomes of average eutherian mammalian group.

\section{Conclusions}

High-resolution X chromosome maps of cetartiodactyl species provide unique information about evolution of intrachromosomal rearrangements. Three conserved syntenic blocks have been identified. We postulate that inversions and centromere repositioning were two key types of rearrangements in course of cetartiodactyl X chromosome evolution. The detailed analysis of the BAC order across multiple species by FISH mapping and bioinformatic analysis allowed the reconstruction of a putative cetartiodactyl ancestral X chromosome. The basal cetartiodactyl group of non-ruminants (pigs, camels, whales, and hippos) share this metacentric ancestral type of $\mathrm{X}$ chromosome. The submetacentric ancestral Ruminantia $X$ chromosome was likely formed by simple centromere shift but it retained the ancestral intrachromosomal structure. Currently observed X chromosome morphological variation was formed by inversions and centromere repositioning during 55 million years of ruminant evolution. Chromosome rearrangements supporting the taxonomic status of ruminant families and subfamilies were found by mapping 26 BAC clones specific to the $\mathrm{X}$ chromosome. The rate of $\mathrm{X}$-specific rearrangements in Ruminantia significantly exceeds that among eutherian mammals. 
Acknowledgments: The work was supported by the Russian Science Foundation (RSF, 16-14-10009). Animal silhouettes were sourced from https:/ / pixabay.com. Preliminary BAC selection and preparation was supported by the United States Department of Agriculture Federal Hatch Project (grant number 538922) and the Biotechnology and Biological Sciences Research Council grants BB/K008226/1 and BB/J010170/1 (to D.M.L). We kindly acknowledge Mary Thompson for establishing cell lines in the Laboratory of Genomic Diversity, NCI-Frederick, MD, USA and Marlys Houck, Julie Fronczek, and Suellen Charter for establishing cell cultures at the San Diego Zoo Institute for Conservation Research's Frozen Zoo. We would like to acknowledge Director of Catoctin Wildlife Preserve and Zoo Richard Hahn. We would like to deeply acknowledge D. Yudkin (IMCB SB RAS), N. Mamaev and E.r Kirillin (Institute of Biological Problems of Cryolithozone SB RAS) for providing muskox sample, A. Sharshov for providing Siberian musk deer and Siberian roe deer samples, G.G. Boeskorov for providing moose sample. We acknowledge P. Dementieva for preparing Siberian roe deer cell line.

Author Contributions: A.G. and A.K. conceived and designed the experiments; A.K., A.M., M.F., D.L. performed BAC clone selection; A.K., J.J., D.L. provide BAC clone material, A.P. performed the experiments and analyzed the data; A.P., A.M. performed bioinformatics analysis; P.P., V.B., O.R., S.O. provided cell lines, M.R., J.B. provided samples, A.P., N.L., P.P., A.K., V.B. prepared suspensions of metaphase chromosome, A.P. wrote the paper. All authors read and approved the final paper.

Conflicts of Interest: The authors declare no conflict of interest.

\section{References}

1. Ohno, S.; Beçak, W.; Beçak, M.L. X-autosome ratio and the behavior pattern of individual X-chromosomes in placental mammals. Chromosoma 1964, 15, 14-30. [CrossRef] [PubMed]

2. Pathak, S.; Stock, A.D. The X chromosomes of mammals: karyological homology as revealed by banding techniques. Genetics 1974, 78, 703-714. [PubMed]

3. Murphy, W.J.; Larkin, D.M.; Everts-Van Der Wind, A.; Bourque, G.; Tesler, G.; Auvil, L.; Beever, J.E.; Chowdhary, B.P.; Galibert, F.; Gatzke, L.; et al. Dynamics of mammalian chromosome evolution inferred from multispecies comparative maps. Science 2005, 309, 613-617. [CrossRef] [PubMed]

4. Graphodatsky, A.S. Conserved and variable elements of mammalian chromosomes. Cytogenet. Anim. 1989, 95-124.

5. Ferguson-Smith, M.A. History and evolution of cytogenetics. Mol. Cytogenet. 2015, 8, 19. [CrossRef] [PubMed]

6. Lee, C.; Griffin, D.K.; O’Brien, P.C.M.; Yang, F.; Lin, C.C.; Ferguson-Smith, M.A. Defining the anatomy of the Rangifer tarandus sex chromosomes. Chromosoma 1998, 107, 61-69. [CrossRef] [PubMed]

7. Huang, L.; Chi, J.; Nie, W.; Wang, J.; Yang, F. Phylogenomics of several deer species revealed by comparative chromosome painting with Chinese muntjac paints. Genetica 2006, 127, 25-33. [CrossRef] [PubMed]

8. Kulemzina, A.I.; Trifonov, V.A.; Perelman, P.L.; Rubtsova, N.V.; Volobuev, V.; Ferguson-Smith, M.A.; Stanyon, R.; Yang, F.; Graphodatsky, A.S. Cross-species chromosome painting in Cetartiodactyla: Reconstructing the karyotype evolution in key phylogenetic lineages. Chromosome Res. 2009, 17, 419-436. [CrossRef] [PubMed]

9. Kulemzina, A.I.; Yang, F.; Trifonov, V.A.; Ryder, O.A.; Ferguson-Smith, M.A.; Graphodatsky, A.S. Chromosome painting in Tragulidae facilitates the reconstruction of Ruminantia ancestral karyotype. Chromosome Res. 2011, 19, 531. [CrossRef] [PubMed]

10. Kulemzina, A.I.; Perelman, P.L.; Grafodatskaya, D.A.; Nguyen, T.T.; Thompson, M.; Roelke-Parker, M.E.; Graphodatsky, A.S. Comparative chromosome painting of pronghorn (Antilocapra americana) and saola (Pseudoryx nghetinhensis) karyotypes with human and dromedary camel probes. BMC Genet. 2014, 15, 68. [CrossRef] [PubMed]

11. Kulemzina, A.I.; Proskuryakova, A.A.; Beklemisheva, V.R.; Lemskaya, N.A.; Perelman, P.L.; Graphodatsky, A.S. Comparative Chromosome Map and Heterochromatin Features of the Gray Whale Karyotype (Cetacea). Cytogenet. Genome Res. 2016, 148, 25-34. [CrossRef] [PubMed]

12. Robinson, T.J.; Harrison, W.R.; Ponce de Leon, F.A.; Davis, S.K.; Elder, F.F.B. A molecular cytogenetic analysis of $\mathrm{X}$ chromosome repatterning in the Bovidae: transpositions, inversions, and phylogenetic inference. Cytogenet. Genome Res. 1998, 80, 179-184. [CrossRef]

13. Rubes, J.; Musilova, P.; Kopecna, O.; Kubickova, S.; Cernohorska, H.; Kulemsina, A.I. Comparative molecular cytogenetics in Cetartiodactyla. Cytogenet. Genome Res. 2012, 137, 194-207. [CrossRef] [PubMed] 
14. Stanyon, R.; Archidiacono, N.; Rocchi, M. Comparative Primate Molecular Cytogenetics: Revealing Ancestral Genomes, Marker Order, and Evolutionary New Centromeres. In Post-Genome Biology of Primates; Hirai, H., Imai, H., Go, Y., Eds.; Springer: Tokyo, Japan, 2012; pp. 193-216. ISBN 978-4-431-54010-6.

15. Chiatante, G.; Capozzi, O.; Svartman, M.; Perelman, P.; Centrone, L.; Romanenko, S.S.; Ishida, T.; Valeri, M.; Roelke-Parker, M.E.; Stanyon, R. Centromere repositioning explains fundamental number variability in the New World monkey genus Saimiri. Chromosoma 2016, 1-11. [CrossRef] [PubMed]

16. Trifonov, V.A.; Kosyakova, N.; Romanenko, S.A.; Stanyon, R.; Graphodatsky, A.S.; Liehr, T. New insights into the karyotypic evolution in muroid rodents revealed by multicolor banding applying murine probes. Chromosome Res. 2010, 18, 265-275. [CrossRef] [PubMed]

17. Trifonov, V.A.; Musilova, P.; Kulemsina, A.I. Chromosome evolution in Perissodactyla. Cytogenet. Genome Res. 2012, 137, 208-217. [CrossRef] [PubMed]

18. Hassanane, M.S.; Chaudhary, R.; Chowdhary, B.P. Microdissected bovine X chromosome segment delineates homoeologous chromosomal regions in sheep, goat and buffalo. Chromosome Res. 1998, 6, 213-217. [CrossRef] [PubMed]

19. Piumi, F.; Schibler, L.; Vaiman, D.; Oustry, A.; Cribiu, E.P. Comparative cytogenetic mapping reveals chromosome rearrangements between the $\mathrm{X}$ chromosomes of two closely related mammalian species (cattle and goats). Cytogenet. Genome Res. 1998, 81, 36-41. [CrossRef]

20. Iannuzzi, L.; Di Meo, G.P.; Perucatti, A.; Incarnato, D.; Schibler, L.; Cribiu, E.P. Comparative FISH mapping of bovid $\mathrm{X}$ chromosomes reveals homologies and divergences between the subfamilies Bovinae and Caprinae. Cytogenet. Genome Res. 2000, 89, 171-176. [CrossRef]

21. Iannuzzi, L.; King, W.A.; Di Berardino, D. Chromosome evolution in domestic bovids as revealed by chromosome banding and FISH-mapping techniques. Cytogenet. Genome Res. 2009, 126, 49-62. [CrossRef] [PubMed]

22. Perucatti, A.; Genualdo, V.; Iannuzzi, A.; Rebl, A.; Di Berardino, D.; Goldammer, T.; Iannuzzi, L. Advanced comparative cytogenetic analysis of $\mathrm{X}$ chromosomes in river buffalo, cattle, sheep, and human. Chromosome Res. 2012, 20, 413-425. [CrossRef] [PubMed]

23. Gallagher, D.S.; Davis, S.K.; De Donato, M.; Burzlaff, J.D.; Womack, J.E.; Taylor, J.F.; Kumamoto, A.T. A Molecular Cytogenetic Analysis of the Tribe Bovini (Artiodactyla: Bovidae: Bovinae) with an Emphasis on Sex Chromosome Morphology and NOR Distribution. Chromosome Res. 1999, 7, 481-492. [CrossRef] [PubMed]

24. Cernohorska, H.; Kubickova, S.; Kopecna, O.; Kulemzina, A.I.; Perelman, P.L.; Elder, F.F.; Robinson, T.J.; Graphodatsky, A.S.; Rubes, J. Molecular cytogenetic insights to the phylogenetic affinities of the giraffe (Giraffa camelopardalis) and pronghorn (Antilocapra americana). Chromosome Res. 2013, 21, 447-460. [CrossRef] [PubMed]

25. Fröhlich, J.; Kubickova, S.; Musilova, P.; Cernohorska, H.; Muskova, H.; Rubes, J. A Comparative Study of Pygmy Hippopotamus (Choeropsis liberiensis) Karyotype by Cross-Species Chromosome Painting. J. Mamm. Evol. 2016, 1-10. [CrossRef]

26. Yang, F.; Graphodatsky, A.S. Animal probes and ZOO-FISH. In Fluorescence in Situ Hybridization (FISH); Liehr, T., Ed.; Springer: Berlin, Germany, 2017; pp. 323-346.

27. Seabright, M. A rapid banding technique for human chromosomes. Lancet 1971, 2, 971-972. [CrossRef]

28. Kent, W.J.; Sugnet, C.W.; Furey, T.S.; Roskin, K.M.; Pringle, T.H.; Zahler, A.M.; Haussler, D. The human genome browser at UCSC. Genome Res. 2002, 12, 996-1006. [CrossRef] [PubMed]

29. Karolchik, D.; Hinrichs, A.S.; Furey, T.S.; Roskin, K.M.; Sugnet, C.W.; Haussler, D.; Kent, W.J. The UCSC Table Browser data retrieval tool. Nucleic Acids Res. 2004, 32, D493-D496. [CrossRef] [PubMed]

30. Pollard, K.S.; Hubisz, M.J.; Rosenbloom, K.R.; Siepel, A. Detection of nonneutral substitution rates on mammalian phylogenies. Genome Res. 2010, 20, 110-121. [CrossRef] [PubMed]

31. Siepel, A.; Bejerano, G.; Pedersen, J.S.; Hinrichs, A.S.; Hou, M.; Rosenbloom, K.; Clawson, H.; Spieth, J.; Hillier, L.W.; Richards, S.; et al. Evolutionarily conserved elements in vertebrate, insect, worm, and yeast genomes. Genome Res. 2005, 15, 1034-1050. [CrossRef] [PubMed]

32. Therneau, T.; Atkinson, B.; Ripley, B. Recursive Partitioning and Regression Trees. R Package Version 4.1-10; Mayo Foundation: Rochester, MN, USA, 2015.

33. Proskuryakova, A.A.; Kulemzina, A.I.; Graphodatsky, A.S. Localisation of NOR on sex chromosome of Tragulus javanicus. Cytogenet. Genome Res. 2018, in preparation. 
34. Ohno, S.; Wolf, U.; Atkin, N.B. Evolution from fish to mammals by gene duplication. Hereditas 1968, 59, 169-187. [CrossRef] [PubMed]

35. Raudsepp, T.; Lee, E.-J.; Kata, S.R.; Brinkmeyer, C.; Mickelson, J.R.; Skow, L.C.; Womack, J.E.; Chowdhary, B.P. Exceptional conservation of horse-human gene order on $\mathrm{X}$ chromosome revealed by high-resolution radiation hybrid mapping. Proc. Natl. Acad. Sci. USA 2004, 101, 2386-2391. [CrossRef] [PubMed]

36. Murphy, W.J.; Sun, S.; Chen, Z.-Q.; Pecon-Slattery, J.; O’Brien, S.J. Extensive Conservation of Sex chromosome Organization Between Cat and Human Revealed by Parallel Radiation Hybrid Mapping. Genome Res. 1999, 9, 1223-1230. [CrossRef] [PubMed]

37. Rodríguez Delgado, C.L.; Waters, P.D.; Gilbert, C.; Robinson, T.J.; Graves, J.A.M. Physical mapping of the elephant $X$ chromosome: conservation of gene order over 105 million years. Chromosome Res. 2009, 17, 917-926. [CrossRef] [PubMed]

38. Quilter, C.R.; Blott, S.C.; Mileham, A.J.; Affara, N.A.; Sargent, C.A.; Griffin, D.K. A mapping and evolutionary study of porcine sex chromosome gene. Mamm. Genome 2002, 13, 588-594. [CrossRef] [PubMed]

39. Avila, F.; Baily, M.P.; Perelman, P.; Das, P.J.; Pontius, J.; Chowdhary, R.; Owens, E.; Johnson, W.E.; Merriwether, D.A.; Raudsepp, T. A Comprehensive Whole-Genome Integrated Cytogenetic Map for the Alpaca (Lama pacos). Cytogenet. Genome Res. 2014, 144, 196-207. [CrossRef] [PubMed]

40. Adega, F.; Chaves, R.; Guedes-Pinto, H. Chromosomal evolution and phylogenetic analyses in Tayassu pecari and Pecari tajacu (Tayassuidae): tales from constitutive heterochromatin. J. Genet. 2007, 86, 19-26. [CrossRef] [PubMed]

41. Bianchi, N.O.; Larramendy, M.L.; Bianchi, M.S.; Cortes, L. Karyological conservatism in South American camelids. Cell. Mol. Life Sci. 1986, 42, 622-624. [CrossRef]

42. Bunch, T.D.; Foote, W.C.; Maciulis, A. Chromosome banding pattern homologies and NORs for the Bactrian camel, guanaco, and llama. J. Hered. 1985, 76, 115-118. [CrossRef]

43. Balmus, G.; Trifonov, V.A.; Biltueva, L.S.; O’Brien, P.C.; Alkalaeva, E.S.; Fu, B.; Skidmore, J.A.; Allen, T.; Graphodatsky, A.S.; Yang, F.; et al. Cross-species chromosome painting among camel, cattle, pig and human: further insights into the putative Cetartiodactyla ancestral karyotype. Chromosome Res. 2007, 15, 499-514. [CrossRef] [PubMed]

44. Árnason, Ú. Comparative chromosome studies in Cetacea. Hereditas 1974, 77, 1-36. [CrossRef] [PubMed]

45. Murphy, W.J.; Pringle, T.H.; Crider, T.A.; Springer, M.S.; Miller, W. Using genomic data to unravel the root of the placental mammal phylogeny. Genome Res. 2007, 17, 413-421. [CrossRef] [PubMed]

46. Price, S.A.; Bininda-Emonds, O.R.; Gittleman, J.L. A complete phylogeny of the whales, dolphins and even-toed hoofed mammals (Cetartiodactyla). Biol. Rev. 2005, 80, 445-473. [CrossRef] [PubMed]

47. Hassanin, A.; Delsuc, F.; Ropiquet, A.; Hammer, C.; van Vuuren, B.J.; Matthee, C.; Ruiz-Garcia, M.; Catzeflis, F.; Areskoug, V.; Nguyen, T.T.; et al. Pattern and timing of diversification of Cetartiodactyla (Mammalia, Laurasiatheria), as revealed by a comprehensive analysis of mitochondrial genomes. C. R. Biol. 2012, 335, 32-50. [CrossRef] [PubMed]

48. O’Brien, S.J.; Menninger, J.C.; Nash, W.G. Atlas of Mammalian Chromosomes; John Wiley \& Sons: Hoboken, NJ, USA, 2006.

49. Chi, J.; Fu, B.; Nie, W.; Wang, J.; Graphodatsky, A.S.; Yang, F. New insights into the karyotypic relationships of Chinese muntjac (Muntiacus reevesi), forest musk deer (Moschus berezovskii) and gayal (Bos frontalis). Cytogenet. Genome Res. 2005, 108, 310-316. [CrossRef] [PubMed]

50. Yang, F.; Müller, S.; Just, R.; Ferguson-Smith, M.A.; Wienberg, J. Comparative chromosome painting in mammals: human and the Indian muntjac (Muntiacus muntjak vaginalis). Genomics 1997, 39, 396-401. [CrossRef] [PubMed]

51. Yang, F.; O’Brien, P.C. M.; Wienberg, J.; Ferguson-Smith, M.A. Evolution of the black muntjac (Muntiacus crinifrons) karyotype revealed by comparative chromosome painting. Cytogenet. Genome Res. 1997, 76, 159-163. [CrossRef]

52. Yang, F.; O’Brien, P.C. M.; Wienberg, J.; Neitzel, H.; Lin, C.C.; Ferguson-Smith, M.A. Chromosomal evolution of the Chinese muntjac (Muntiacus reevesi). Chromosoma 1997, 106, 37-43. [CrossRef] [PubMed]

53. Kingdon, J. The Kingdon Field Guide to African Mammals; Bloomsbury Publishing: London, UK, 2015.

54. Buckland, R.A.; Evans, H.J. Cytogenetic aspects of phylogeny in the Bovidae. Cytogenet. Genome Res. 1978, 21, 42-63. [CrossRef] 
55. Vozdova, M.; Ruiz-Herrera, A.; Fernandez, J.; Cernohorska, H.; Frohlich, J.; Sebestova, H.; Kubickova, S.; Rubes, J. Meiotic behaviour of evolutionary sex-autosome translocations in Bovidae. Chromosome Res. 2016, 24, 325-338. [CrossRef] [PubMed]

56. Iannuzzi, L. Standard karyotype of the river buffalo (Bubalus bubalis L., $2 n=50$ ). Report of the committee for the standardization of banded karyotypes of the river buffalo. Cytogenet. Cell Genet. 1994, 67, 102-113. [CrossRef] [PubMed]

57. Hsu, T.C.; Benirschke, K. An atlas of Mammalian Chromosomes; Springer Science \& Business Media: Berlin, Germany, 2013; Volume 10.

58. Kingswood, S.C.; Kumamoto, A.T. Madoqua kirkii. Mamm. Species 1997, 1-10. [CrossRef]

59. Pevzner, P.; Tesler, G. Human and mouse genomic sequences reveal extensive breakpoint reuse in mammalian evolution. Proc. Natl. Acad. Sci. USA 2003, 100, 7672-7677. [CrossRef] [PubMed]

60. Larkin, D.M.; Pape, G.; Donthu, R.; Auvil, L.; Welge, M.; Lewin, H.A. Breakpoint regions and homologous synteny blocks in chromosomes have different evolutionary histories. Genome Res. 2009, 19, 770-777. [CrossRef] [PubMed]

61. Rubtsov, N.B.; Rubtsova, N.V.; Anopriyenko, O.V.; Karamysheva, T.V.; Shevchenko, A.I.; Mazurok, N.A.; Nesterova, T.B.; Zakian, S.M. Reorganization of the $\mathrm{X}$ chromosome in voles of the genus Microtus. Cytogenet. Genome Res. 2002, 99, 323-329. [CrossRef] [PubMed]

62. Kobayashi, T.; Yamada, F.; Hashimoto, T.; Abe, S.; Matsuda, Y.; Kuroiwa, A. Centromere repositioning in the $\mathrm{X}$ chromosome of $\mathrm{XO} / \mathrm{XO}$ mammals, Ryukyu spiny rat. Chromosome Res. 2008, 16, 587-593. [CrossRef] [PubMed]

63. Gladkikh, O.L.; Romanenko, S.A.; Lemskaya, N.A.; Serdyukova, N.A.; O’Brien, P.C.; Kovalskaya, J.M.; Smorkatcheva, A.V.; Golenishchev, F.N.; Perelman, P.L.; Trifonov, V.A.; et al. Rapid Karyotype Evolution in Lasiopodomys Involved at Least Two Autosome-Sex Chromosome Translocations. PLoS ONE 2016, 11, e0167653. [CrossRef] [PubMed]

64. Kim, H.; Lee, T.; Sung, S.; Lee, C.; Kim, H. Reanalysis of Ohno's hypothesis on conservation of the size of the $\mathrm{X}$ chromosome in mammals. Anim. Cells Syst. 2012, 16, 438-446. [CrossRef]

65. Murphy, W.J.; Stanyon, R.; O'Brien, S.J. Evolution of mammalian genome organization inferred from comparative gene mapping. Genome Biol. 2001, 2, reviews0005.1-reviews0005.8. [CrossRef] 